Volume $50 \cdot$ Number $2 \cdot 2013$

\title{
COMMENTARY ON BIOENGINEERING OF WHEELCHAIRS: THE PAST 50 YEARS
}

RORY A. COOPER, PHD

$\mathrm{t}$ was a pleasure to become reacquainted with the landmark article "Bioengineering methods of wheelchair evaluation" by Peizer et al. from the 1964 Bulletin of Prosthetics Research. As is to be expected, in some areas, there have been tremendous advances since the article was written and, in others, our understanding remains limited. Probably the three most profound aspects of the Peizer et al. article are that (1) much of the wheelchair engineering research at the time could be summarized in a single article, (2) the Department of Veterans Affairs Prosthetics Center was on the leading edge of multidisciplinary team research, and (3) far fewer people were conducting wheelchair-related research. The breadth of the research described by Peizer et al. is remarkable for its time, but the depth of the knowledge was much less than we know today.

Most would agree that the greatest changes that have taken place over the past 50 years are in the areas of ability to image the upper limbs; biomechanics (kinematics, especially kinetics) of wheelchair propulsion and transfers; design of wheelchairs from the materials, electronics, software, and fabrication to the style; and human-to-wheelchair interfaces for both manual and power chairs.
The most profound difference is that the wheelchairs of today are vastly superior to the wheelchairs of 50 years ago. At the time of publication of Peizer et al.'s article, there was one dominant manufacturer and very few options to include sizes. People could literally memorize the entire catalog. Today, there are more than 100 companies and thousands of models with literally hundreds of thousands of options.

Manual wheelchairs are much lighter, efficient, ergonomic, and socially acceptable. A growing number of clinicians understand the need for matching the design and configuration closely to the needs and abilities of the user. Electric powered wheelchairs (EPWs) share very little in common with their ancestors of 50 years ago. EPWs today have computeroperated interfaces and power controls that were unthinkable at that time. Further, the EPW seat and chassis systems have been separated to be more like a car than a "wheeled chair." EPW chassis often have independent suspension, and seats can range from simple "captain chairs" to fully articulated with power elevation, leg-rest angle, tilt, and recline. Fifty years ago, sports wheelchairs (e.g., racing, rugby, basketball, and hand-cycling) did not exist. Athletes competed in essentially the same chairs that they used everyday. Now, nearly every adaptive sport uses custom-designed wheelchairs and athletic performances are astoundingly better than 50 years ago. People who use wheelchairs are healthier and live longer because science has led to better understanding of the benefits of exercise, adaptive sports and recreation, nutrition, smoking cessation, upper-limb preservation, pressure ulcer prevention, and skills training.

A number of barriers still remain. Although international wheelchair standards exist, they are seldom used in decision making or uniformly applied, leading to high variability in the quality, efficacy, and safety of wheelchairs. Reimbursement policies still vary widely, and the majority of insurers still severely limit access to appropriate quality and functioning wheelchairs. There remain millions of people without access to wheelchairs or the ability to operate current wheelchairs. There remains much to be learned about designing optimal wheelchairs, preventing secondary conditions, matching wheelchairs to user needs, evaluating the interaction of wheelchairs with the built environment, and infusing that knowledge into user and clinical communities. 